\title{
A PARALLEL PARTITIONED APPROACH ON FLUID-STRUCTURE INTERACTION SIMULATION USING THE MULTISCALE UNIVERSAL INTERFACE COUPLING LIBRARY
}

\section{WENDI LIU, WEI WANG, ALEX SKILLEN, STEPHEN M. LONGSHAW, CHARLES MOULINEC AND DAVID R. EMERSON}

Scientific Computing Department, Science and Technology Facilities Council, Warrington, UK

Key words: Fluid-Structure Interaction, Multiscale Universal Interface Coupling Library, Partitioned Approach, Hydrofoil.

\begin{abstract}
Fluid-Structure Interaction (FSI) is a phenomenon that appears in a wide range of scientific research and engineering applications at different spatial and temporal scales. There are many in-house/commercial solvers capable of modelling FSI, but high numerical robustness and high scalability codes are still in demand. In this study, a numerical framework for FSI simulations has been developed using a partitioned approach aimed at both high numerical robustness and good computational scalability. Open-source software is used for each component of the coupled solution, with OpenFOAM and FEniCS adopted to simulate the computational fluid dynamics and computational structural mechanics, respectively. A coupling interface between the fluid and structural computational domains is realised using the open-source Multiscale Universal Interface (MUI) scientific code coupling library. To achieve a tight and stable coupling, various FSI coupling algorithms have been implemented in the MUI. The behaviour of this framework has been assessed for simulations of a blunt trailing edge hydrofoil at different working conditions with vortex-shedding induced vibration.
\end{abstract}

\section{INTRODUCTION}

Fluid-Structure Interaction (FSI) is the two-way coupling between fluid flow and structural response. This phenomenon occurs in a wide range of scientific research and engineering applications at different spatial and temporal scales. Examples include the hydroelasticity of a hydrofoil, the aeroelasticity of turbine blades and the flow of blood in an artery $[1,2]$. To accurately and efficiently tackle large-scale FSI problems involving billions of degrees of freedom, such a tool needs to show good scalability. Robustness and stability are also required for a strong FSI coupling.

The numerical procedures for solving FSI problems can be categorised into two approaches: the monolithic approach and the partitioned approach. A monolithic approach considers the fluid dynamics and structural mechanics in the same mathematical framework to form a single system for the entire problem, in which the fluid and structural domains are solved simultaneously by a unified algorithm. The interfacial conditions between these two domains are implicit in the solution procedure [3]. This approach can achieve stable and accelerated convergence for strongly coupled systems (where the structural response is highly dependent upon the fluid forces, and vice-versa), but it can be increasingly difficult to cope with FSI 
applications not originally considered, such as involving additional physical domain and phase of fluid. On the other hand, the partitioned approach treats the fluid and the structure as two computational domains which can be solved separately with their respective discretisations and numerical algorithms. The interfacial conditions are explicit, with information being communicated between domains. A partitioned approach for multi-physics coupling has some advantages over a monolithic one since it is possible to adopt existing codes with different discretisation types and algorithmic implementations. One of key challenges in a partitioned approach is to develop an efficient interface between domains, which is robust, highly scalable and with low overhead, in parallel computing.

In this study, we developed an effective and robust methodology for FSI simulations that aims for good scalability using the partitioned approach to allow flexibility and methodological extensibility. The developed approach has been validated in a series of cases, such as 1-D heat transfer, 2-D flow over an elastic plate behind a rigid cylinder, 3-D channel flow over elastic beams of different elasticity. This paper presents the more complex and practical case of the simulation of the combined fluid dynamics and structural mechanics of an elastic hydrofoil under different flow regimes.

This paper is organised as follows: The solvers and governing equations are presented in Section 2, details of validations and a preliminary scalability test of the FSI framework in Section 3 then simulation details and results of the test case of vortex-shedding induced vibration of a hydrofoil with blunt trailing edge in Section 4. Finally, conclusions are drawn and future directions are discussed in Section 5

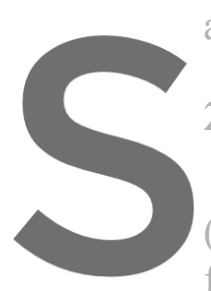

\section{SOIVERS AND}

OpenFOAM [4] and

(CFD) and Computatio

fluid and structural domains is
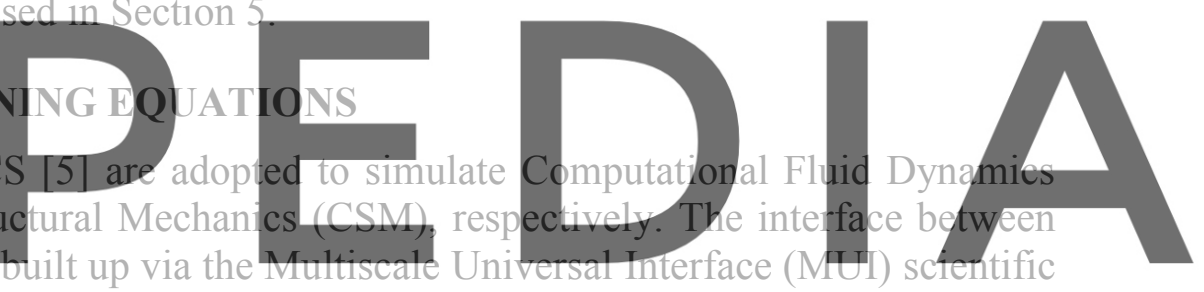

code coupling library [6]. This library has been demonstrated to provide good scalability [7]

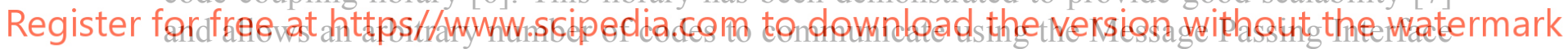

(MPI) standard, via a cloud of points. Two FSI coupling algorithms (Fixed Relaxation and Aitken's $\delta^{2}$ ) have been developed to achieve a tight and stable coupling.

\subsection{Fluid solver}

In the present framework, a new CFD solver pimpleFSIFoam is developed based on the standard OpenFOAM solver pimpleFoam for the fluid domain using the finite volume method. The solver inherits the same key algorithms from pimpleFoam to solve steady and/or unsteady incompressible fluid simulation. The Reynolds Averaged Navier-Stokes (RANS) equations for incompressible transient Newtonian fluid with Arbitrary Lagrange-Euler (ALE) formulation to tackle moving boundary conditions are listed below.

$$
\begin{gathered}
\nabla \cdot\left(\boldsymbol{U}-\boldsymbol{U}_{s}\right)=0, \\
\frac{\partial \boldsymbol{U}}{\partial t}+\nabla \cdot\left[\left(\boldsymbol{U}-\boldsymbol{U}_{s}\right) \boldsymbol{U}\right]+\frac{1}{\rho_{f}} \nabla p-\nabla \cdot\left(v_{f} \nabla \boldsymbol{U}\right)-g=0,
\end{gathered}
$$

where $\boldsymbol{U}$ is the fluid velocity, $\boldsymbol{U}_{\boldsymbol{s}}$ is the grid velocity, $t$ is the time, $\rho_{f}$ is the fluid density, $p$ is the fluid pressure, $g$ is the gravational acceleration, $v_{f}$ is the effective kinematic viscosity of the fluid that is the combination of laminar kinematic viscosity and turbulent kinematic viscosity. The pressure-momentum coupling algorithm, PIMPLE, which is the merge of 
Pressure Implicit Split Operator (PISO) and Semi-Implicit Method of Pressure Linked Equations (SIMPLE), is used for pressure-velocity coupling [8]. Grid velocities of the control volume at the fluid-structure interface are determined at runtime according to the results of the structure solver. The other grid velocities of the fluid domain are determined by solving a Laplace equation [9]. Additional functions have been added to the solver to: a) calculate the fluid forces on each computational grid that is located in the interface between the domains and send the forces to the structural solver; $b$ ) receive the displacement of each computational grid point handled by the interface and apply the mesh movement accordingly; c) conduct subiterations for fluid-structure interaction at each time step of fluid simulation. Section 2.3 will discuss these additional functions in detail.

\subsection{Structural solver}

The in-house CSM solver is developed based on the FEniCS library using the finite element method. The elastodynamics formulation with the force balance of linear momentum is written as

$$
\rho_{s} \frac{\partial^{2} \boldsymbol{d}}{\partial t^{2}}=\nabla \cdot\left(J \sigma_{s} \boldsymbol{F}^{-T}\right)+\rho_{s} \boldsymbol{f}_{s}
$$

where $\rho_{S}$ is the density of the structure, the vector $\boldsymbol{d}$ is the structural displacement and $\boldsymbol{f}_{S}$ expresses the exterior body forces being exerted on the structure. The gradient of the deformation $\boldsymbol{F}$ is calculated with the identity matrix $\boldsymbol{I}$ as

$$
F=I+\nabla d
$$

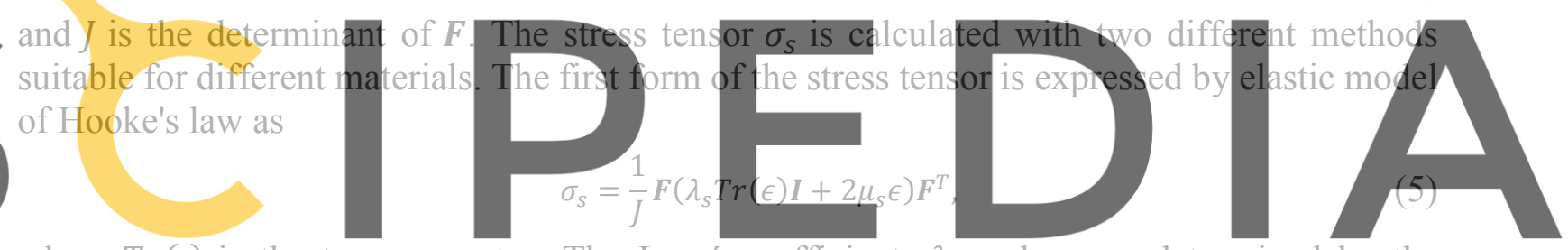

where $\operatorname{Tr}(\cdot)$ is the trace operator. The Lamé coefficients $\lambda_{s}$ and $\mu_{s}$ are determined by the

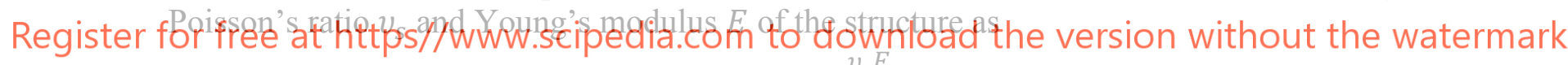

$$
\begin{gathered}
\lambda_{S}=\frac{v_{S} E}{\left(1+v_{S}\right)\left(1-2 v_{S}\right)^{\prime}} \\
\mu_{s}=\frac{E}{2\left(1+v_{s}\right)^{\prime}},
\end{gathered}
$$

The strain tensor $\epsilon$ is expressed as

$$
\epsilon=\frac{1}{2}\left(\nabla \boldsymbol{d}+(\nabla \boldsymbol{d})^{T}\right)
$$

The second form of the stress tensor is expressed by the hyper-elastic St. Vernant-Kirchhoff model as

$$
\sigma_{s}=\frac{1}{J} \boldsymbol{F}\left(\lambda_{s} \operatorname{Tr}(\boldsymbol{G}) \boldsymbol{I}+2 \mu_{s} \boldsymbol{G}\right) \boldsymbol{F}^{T},
$$

where $\boldsymbol{G}$ is the Green Lagrangian strain tensor that is determined by

$$
\boldsymbol{G}=\frac{1}{2}(\boldsymbol{C}-\boldsymbol{I})
$$

The right Cauchy-Green strain tensor $\boldsymbol{C}$ is calculated as

$$
C=F F^{T} \text {. }
$$


Hooke's law is limited to small deformations of the structure, while the St. Vernant-Kirchhoff model is able to handle large deformations but remains valid for small strains $[10,11]$. The onestep $\Theta$ scheme is implemented for time-stepping of both Hooke's law and the St. VernantKirchhoff model. This enables a clean switch between the second-order Crank-Nicholson scheme, the first-order backward-Euler scheme or the first-order forward-Euler scheme by setting the value of $\Theta$ between 0 and 1 .

In addition to the aforementioned CSM solver, we have also implemented an alternative solver as follows. The elastodynamics formulation can alternatively be expressed in the form of a generalized $n$-degree of freedom (DOF) harmonic oscillator equation as

$$
\boldsymbol{M} \frac{\partial^{2} \boldsymbol{d}}{\partial t^{2}}+\boldsymbol{C}_{\boldsymbol{s}} \frac{\partial \boldsymbol{d}}{\partial t}+\boldsymbol{K} \boldsymbol{d}=\boldsymbol{F}(t)
$$

where $\boldsymbol{M}$ is the mass matrix, $\boldsymbol{K}$ is the stiffness matrix and $\boldsymbol{F}$ is the external load, $\boldsymbol{C}_{\boldsymbol{s}}$ is the damping matrix. In the present in-house structural solver, the damping matrix is modelled based on Rayleigh damping as

$$
\boldsymbol{C}_{s}=\alpha_{m} \boldsymbol{M}+\alpha_{k} \boldsymbol{K}
$$

where $\alpha_{m}$ and $\alpha_{k}$ are Rayleigh damping parameters and are determined on a case-by-case basis. Combining Eq (12) and (13) gives

$$
\boldsymbol{M} \frac{\partial^{2} \boldsymbol{d}}{\partial t^{2}}+\left(\alpha_{m} \boldsymbol{M}+\alpha_{k} \boldsymbol{K}\right) \frac{\partial \boldsymbol{d}}{\partial t}+\boldsymbol{K} \boldsymbol{d}=\boldsymbol{F}(t) .
$$

For time stepping, the generalised- $\alpha$ method, which is an extension of the Newmark- $\beta$ method is used to achieye a seco oscillator equation is al cases show that with generalized $n-\mathrm{DOF}$ harmonic Hooke's law using the p PE

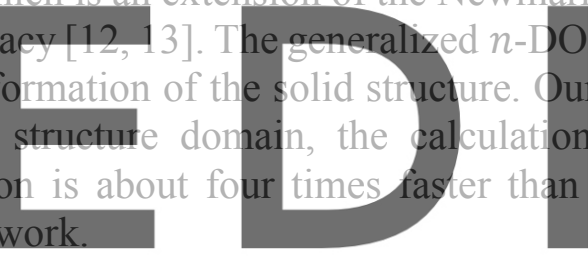
OF har
on vali
on that

2.3 Fluid-structure interaction.

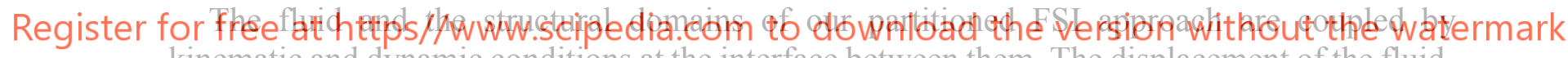
kinematic and dynamic conditions at the interface between them. The displacement of the fluidstructure interface must be consistent from one domain to another

$$
d_{s}=d_{f}
$$

where $\boldsymbol{d}_{\boldsymbol{s}}$ and $\boldsymbol{d}_{\boldsymbol{f}}$ represent the displacement at the fluid-structure interface in the structure and fluid domains, respectively. When the displacement of the structure domain at the interface has been determined by the structural solver, it is applied to the fluid domain as a moving Dirichlet boundary condition for the Arbitrary Lagrangian-Eulerian method for Navier-Stokes equations. The fluid forces, or tractions, acting on the structural domain via the interface according to the elastodynamics formulations have to follow the dynamic condition:

$$
\sigma_{s} \cdot \boldsymbol{n}_{\boldsymbol{s}}=\boldsymbol{t}_{\boldsymbol{f}}
$$

where $\boldsymbol{n}_{\boldsymbol{s}}$ is the outward surface normal unit vector of a solid cell and $\boldsymbol{t}_{\boldsymbol{f}}$ is the traction at the fluid-structure interface, which is calculated as

$$
\boldsymbol{t}_{\boldsymbol{f}}=\sigma_{f} \cdot \boldsymbol{n}_{\boldsymbol{f}}
$$

where $\boldsymbol{n}_{\boldsymbol{f}}$ is the outward surface normal unit vector of a fluid control volume. The stress tensor at the interface $\sigma_{f}$, which is calculated from the fluid domain with an incompressible Newtonian 
fluid is expressed as

$$
\sigma_{f}=-p \boldsymbol{I}+\tau,
$$

where $p$ is the pressure force and $\tau$ is the viscous component of the stress tensor that is calculated as

$$
\tau=\mu_{f}\left(\nabla \boldsymbol{U}+\nabla \boldsymbol{U}^{T}\right) .
$$

This dynamic condition dictates that the forces acting on the fluid-structure interface have to be consistent between the two domains. When the generalized $n$-DOF harmonic oscillator equation is used, the forces at the interface are applied to the structural domain in the form of a Dirichlet boundary condition as the right-hand term of $\mathrm{Eq}$ (14).

It can be readily shown that for a partitioned approach, a loose coupling between fluid and solid domains has the potential to be unconditionally unstable irrespective of the time-step size for certain classes of FSI problems (such as those where the density ratio between the solid and fluid domains is small) [14]. There are several coupling methods to stabilise the numerical simulation, such as the fixed relaxation and Aitken's $\delta^{2}$ methods, both of which have been implemented in the developed solver through the MUI library. Both implemented methods are achieved through the fixed-point Gauss-Seidel iterative approach. The displacement of the structure at the $(k+1)^{\text {th }}$ iteration, $\boldsymbol{d}_{k+1}$, under the fixed-point Gauss-Seidel iteration approach can be expressed as

$$
d_{k+1}=d_{k}+\omega_{k} R_{k}
$$

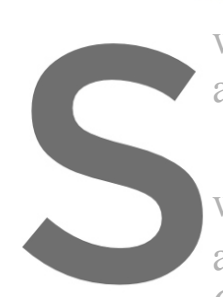

where $\omega_{k}$ is the under

at the $k^{\text {th }}$ iteration, $R_{l}$

where $F_{s}$ and $F_{f}$ are the

a composite function of
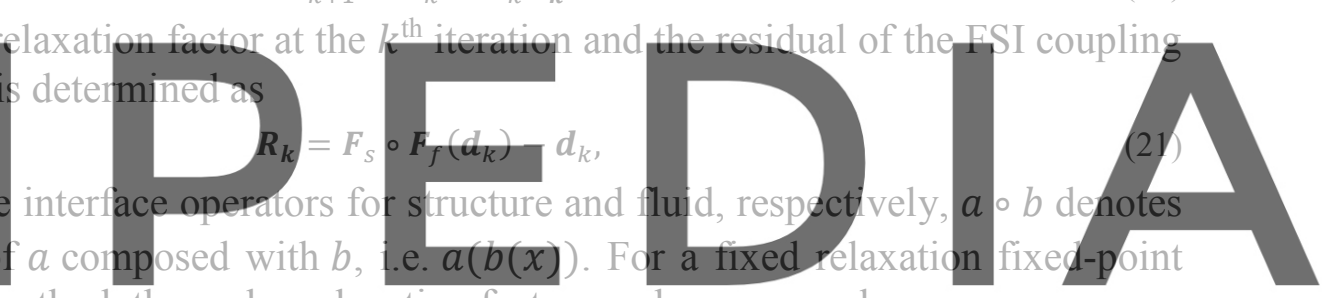

Gauss-Seidel iteration method, the under-relaxation factor can be expressed as

Register for free at https//www.scipedia.com to apstantbad the version without the watermark

For the Aitken's $\delta^{2}$ fixed-point Gauss-Seidel iteration method, the under-relaxation factor is calculated as

$$
\omega_{k}=-\omega_{k-1} \frac{\left(\boldsymbol{R}_{k-1}\right)^{T}\left(\boldsymbol{R}_{\boldsymbol{k}}-\boldsymbol{R}_{\boldsymbol{k}-1}\right)}{\left\|\boldsymbol{R}_{\boldsymbol{k}}-\boldsymbol{R}_{\boldsymbol{k}-1}\right\|^{2}} .
$$

A constraint is applied to the Aitken's $\delta^{2}$ relaxation factor for stability as

$$
\omega_{k}=\operatorname{sgn}\left(\omega_{k}\right) \min \left(\left|\omega_{k}\right|, \omega_{\max }\right) \text {, }
$$

where $\omega_{\max }$ is the maximum value of the under relaxation factors between the $1^{\text {st }}$ and the $k^{\text {th }}$ iterations. The fixed relaxation method is easier to implement, but has a slower convergence rate. The Aitken's $\delta^{2}$ method requires slightly more computational resources per sub-iteration than the fixed relaxation method, but has a quicker convergence rate. 


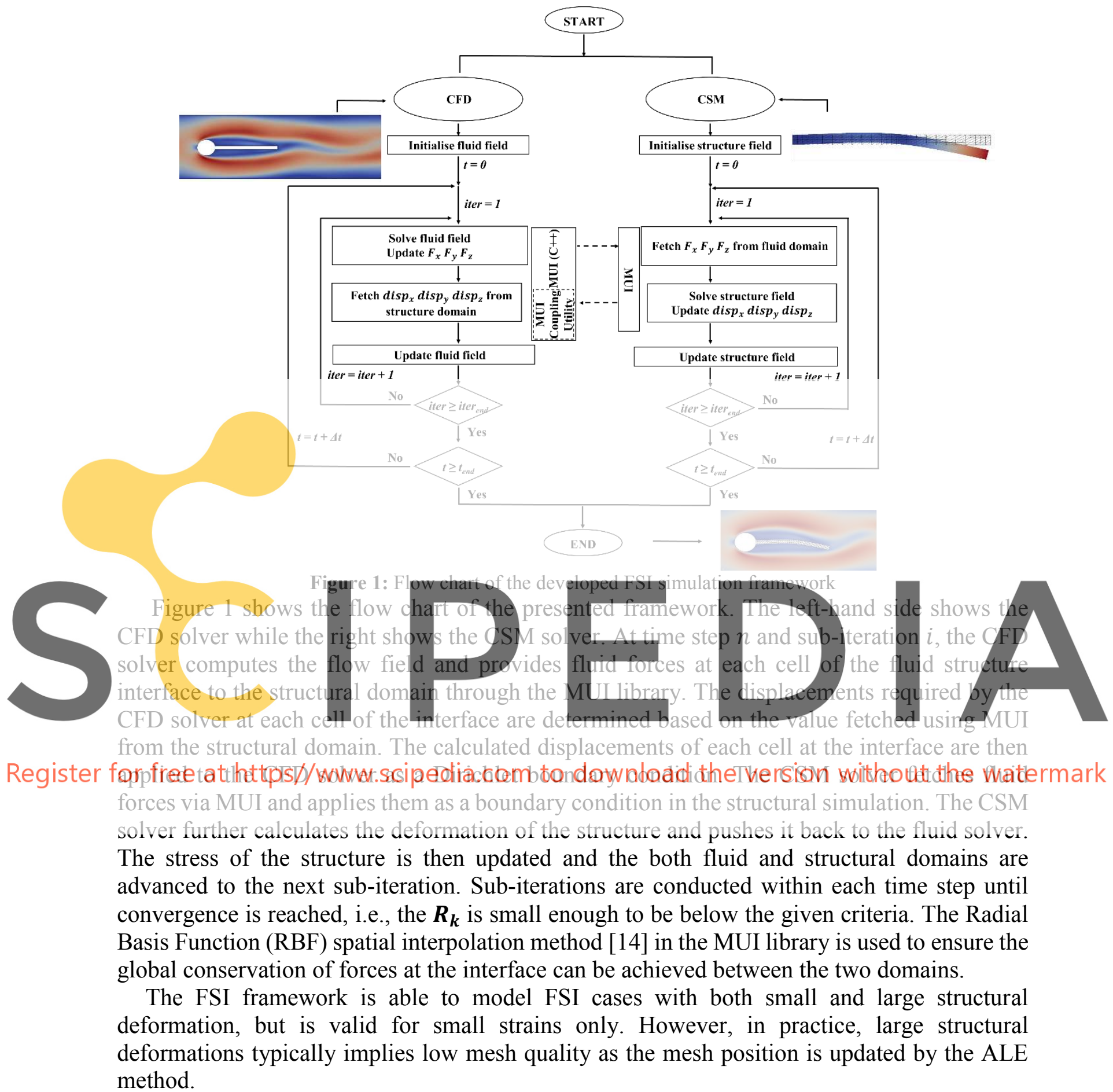

\section{VERIFICATION AND SCALABILITY TESTS}

Simulations have been carried out and compared with published results for the developed FSI framework. Note that verification for each part of the FSI framework, i.e. MUI with FSI coupling algorithms, pimpleFSIFoam and the in-house structure solver using FEniCS, can be 
found in Liu et al. [15].

\subsection{2-D flow over an elastic plate behind a rigid cylinder}

The first benchmark case is a 2-D flow over a rigid circular cylinder with an elastic plate. The geometry and fluid and boundary conditions can be found in Turek and Hron [16]. In the present study, the specific plate considered has a density of $1 \times 10^{3} \mathrm{~kg} / \mathrm{m}^{3}$, a Poisson's ratio of 0.4 and a Young's modulus of $5.6 \times 10^{6} \mathrm{~Pa}$. The Reynolds number based on the maximum velocity and the cylinder diameter is 200 . A total of 25,478 hexahedral cells on fluid domain and 10,143 DOFs on structure domain are employed after a study of grid independence tests.
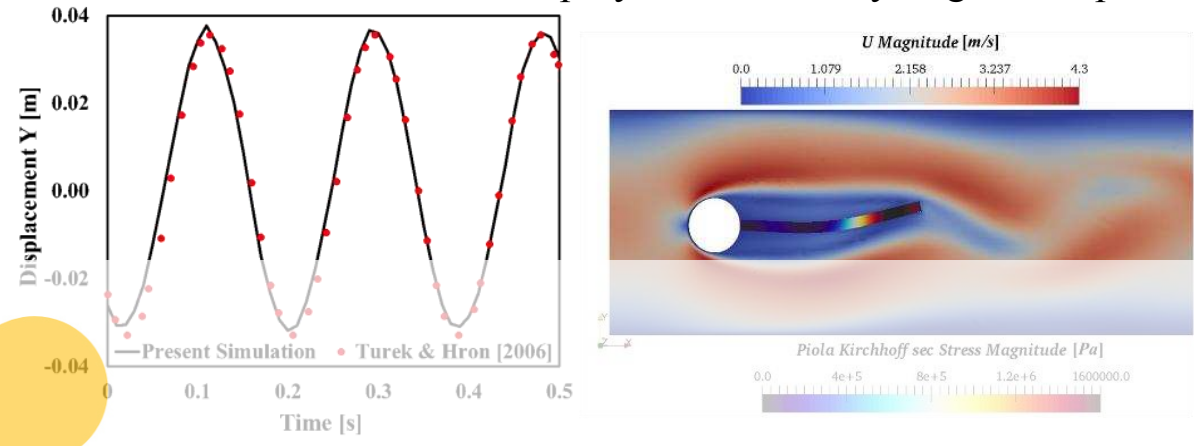

Figure 2: Displacement over time (left) and velocity magnitude contour (right) of flow over elastic plate with rigid cylinder.

Figure 2 shows the tip displacement of the plate, the velocity contour of the fluid domain and the stress distribution in the structural domain. The simulation results show that the tip displacements of the plat qualitatively comparabl 3.2 3-D parabolic flow

Our FSI framework channel flow with a par late agree
le with F $\mathrm{F}$
has also
arabolic vel Figure 3.9 of Slyng
in elastic beam
been tested using
velocity inlet pro
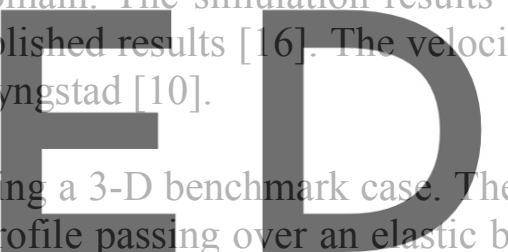

fixed to the lower channel wall. Simulation parameters can be found in [17]. The incoming peak

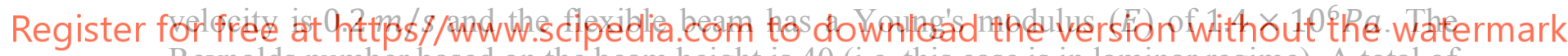

Reynolds number based on the beam height is 40 (i.e. this case is in laminar regime). A total of 933,888 hexahedral cells on fluid domain and 12,152 DOFs on structure domain are employed after a study of grid independence tests. The $x$-axis and $y$-axis displacements $\left(D_{x}\right.$ and $\left.D_{y}\right)$ are taken at a selected monitoring point, as detailed in Tukovic et al. [17] and listed in Table 1, where they are compared with results from Richter [18] and Tukovic et al. [17]. The present simulation has effectively the same $x$-axis displacement as that of Richter [18] while the difference between those in Tukovic et al. [17] are less than 2\%.

Table 1: Displacement of the elastic beam compared with published results

\begin{tabular}{c|cc|cc}
\hline & \multicolumn{2}{|c|}{$E=1.4 \times 10^{6} \mathrm{~Pa}$} & \multicolumn{2}{c}{$E=1 \times 10^{4} \mathrm{~Pa}$} \\
\hline & $D_{x}[\mathrm{~m}]$ & $D_{y}[\mathrm{~m}]$ & $D_{x}[\mathrm{~m}]$ & $D_{y}[\mathrm{~m}]$ \\
\hline Present Simulation & $5.95 \times 10^{-5}$ & $2.36 \times 10^{-5}$ & $1.52 \times 10^{-2}$ & $4.77 \times 10^{-3}$ \\
\hline Tukovic et al.[17] & $5.93 \times 10^{-5}$ & $2.40 \times 10^{-5}$ & $1.46 \times 10^{-2}$ & $5.00 \times 10^{-3}$ \\
\hline Richter [18] & $5.95 \times 10^{-5}$ & - & - & - \\
\hline $\begin{array}{c}\text { Difference fromTukovic } \\
\text { et al. }[17]\end{array}$ & $+0.34 \%$ & $-1.67 \%$ & $+4.11 \%$ & $-4.60 \%$ \\
\hline
\end{tabular}


To demonstrate the ability of the present FSI framework with a particularly challenging FSI case, the simulation of a softer beam is conducted with a peak flow velocity of $0.3 \mathrm{~m} / \mathrm{s}$ and with the Young's Modulus of the beam equal to $1 \times 10^{4} \mathrm{~Pa}$, while all other conditions are kept the same. The $x$-axis and $y$-axis displacements at the selected monitoring point are listed in Table 1 and compared with Tukovic et al. [17]. The difference between the present simulation and that of Tukovic et al. [17] is less than 5\%, which suggests the developed FSI framework behaves as expected while simulating challenging (highly flexible) problems in 3-D.

\subsection{Scalability testing of the FSI framework}

The fully coupled 3-D FSI case discussed in Section 3.2 is chosen here. Tests were achieved by varying the number of CPU cores of the fluid and structural solvers. More specifically, for the first test a fixed value of 100 MPI tasks has been employed for the structural domain while the number for the fluid domain was varied up to 1000 MPI tasks. For our subsequent test, a fixed number of 500 MPI ranks for the fluid domain was employed while up to 1000 were used for the structural domain to test the scaling performance of the FEniCS based in-house structural solver. In order to test the scalability of the framework up to 1000 CPU cores, we employed much larger hexahedral cells ( 83 million) and DOFs (2.5 million) than required by this case in fluid and structure domains, respectively.

As shown in Figure 3, the in-house CFD solver, pimpleFSIFoam, shows good scalability, with parallel efficiency at approximately 68\% for 1000 MPI ranks. However, the parallel efficiency of the in-house structural solver is around 40\% for the same number of MPI ranks. Given the underlying framework used, FEniCS, is noted for its good parallel ability, and the most likely reason for the performance of the structural solver is that the number of DOF was
not large enough to scale in the considered case, causing communications between MPI rapks
to occupy a significant proportion of the computational resources and simulation time. Future
work will be carried out to identify the bottleneck of the overall framework and optimise the
implementation to enhance scalability.

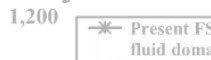

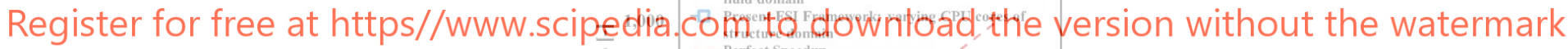

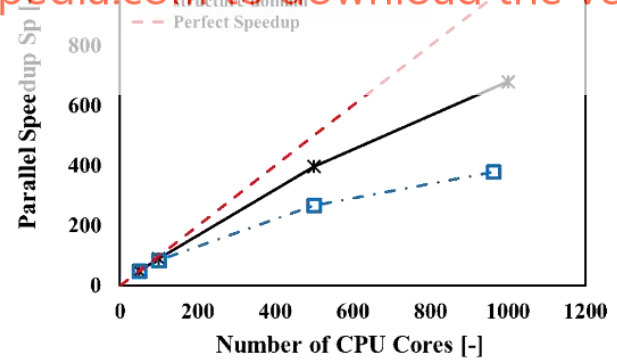

Figure 3: Solvers performance in scalability

\section{VORTEX-SHEDDING INDUCED VIBRATION OF A HYDROFOIL WITH BLUNT TRAILING EDGE}

\subsection{Simulation setup}

Three-dimensional vortex-induced vibration of the trailing edge of a hydrofoil was simulated, following the experimental setup of Ausoni [19]. The NACA0009 hydrofoil is subject to a uniform incoming flow. The hydrofoil mounting can be assumed to embody a perfect embedding on one side and a pivot embedding on the other (see Figure 4). The hydrofoil 
is set to make, zero Angle of Attack (AoA) with the incoming flow. The velocity of the incoming flow is $C_{r e f}$ along $x$-axis direction. The density of the fluid is $998 \mathrm{~kg} / \mathrm{m}^{3}$ and kinetic viscosity is $1 \times 10^{-6} \mathrm{~m}^{2} / \mathrm{s}$. The density of the hydrofoil structure is $7800 \mathrm{~kg} / \mathrm{m}^{3}$. The Young's modulus and Poisson's ratio of the hydrofoil are $2.1 \times 10^{11} \mathrm{~Pa}$ and 0.3 , respectively. The chord length, $L$, is $1 \mathrm{~m}$. The span length, $B$, is $0.15 \mathrm{~m}$. The height of the tripped trailing edge, $h$, is $0.00322 \mathrm{~m}$. The Reynolds's number based on the trailing edge height, $R e_{h}$ is defined as

$$
R e_{h}=\frac{\rho h C_{r e f}}{\mu}
$$

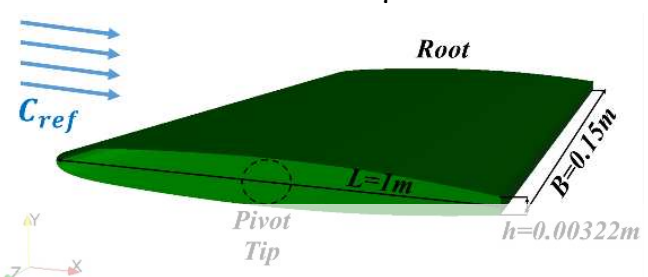

Figure 4: Schematic plot of the hydrofoil case

Following the experimental setup, the fluid domain extends $0.5 \mathrm{~L}$ away from the leading edge of the hydrofoil towards the upstream, $7 \mathrm{~L}$ away from the leading edge towards the downstream and $0.7 \mathrm{~L}$ towards both the maximum and minimum $y$-axis domain boundaries. Both 2-D (i.e. one cell along the spanwise direction in the fluid domain) and 3-D (i.e. 100 cells along the

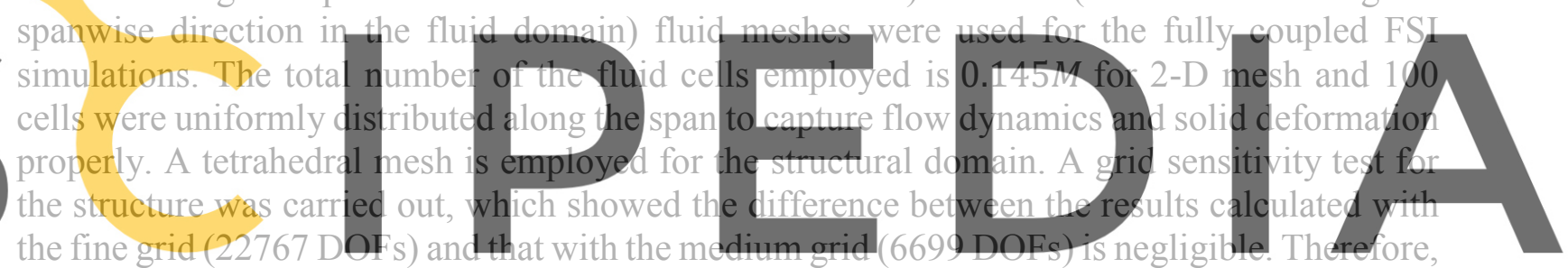
the medium grid with 6699 DOFs is employed. The topology of the fluid and structural grids

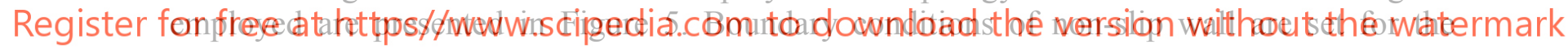
hydrofoil, top and bottom boundaries. The inlet boundary has a uniform fixed value of velocity and zero gradient of pressure. The outlet boundary is pressure outlet boundary condition. The $k-\omega$ turbulence model [20] is used to tackle the turbulence effect.
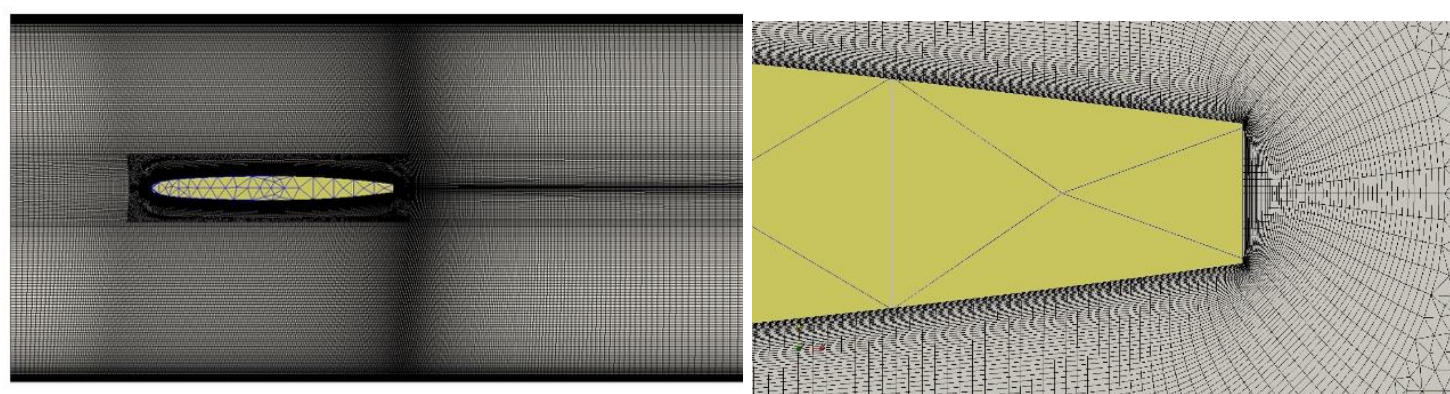

Figure 5: Mesh for both the fluid and structure domains of the hydrofoil case with a zoom-out view of the domain (left) and zoom-in view at the trailing edge of the hydrofoil (right).

The experiments showed that the lock-in regime for the hydrofoil with tripped trailing edge is in the region of $4.80 \times 10^{4} \leq R e_{h} \leq 5.35 \times 10^{4}$. Accordingly, four cases have been simulated using the 2-D fluid mesh, which are $R e_{h}=3.86 \times 10^{4}$ (before the lock-in regime), 
$4.83 \times 10^{4}$ (the beginning of the lock-in regime), $5.31 \times 10^{4}$ (the end of the lock-in regime) and $7.08 \times 10^{4}$ (the lock-off regime). Three cases have been simulated using the 3-D fluid mesh, i.e., $R e_{h}=3.86 \times 10^{4}, 4.83 \times 10^{4}$ and $6.44 \times 10^{4}$.

\subsection{Results of vortex-shedding induced vibration of a hydrofoil}
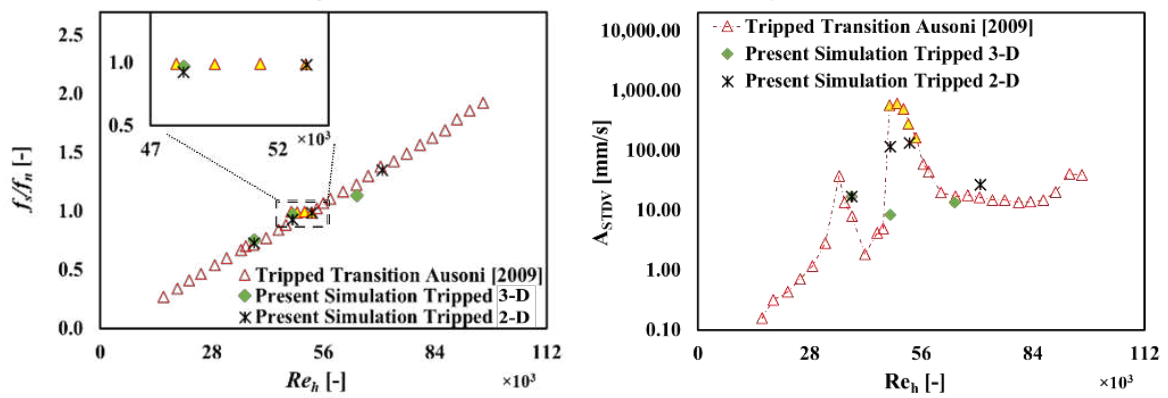

Figure 6: Frequency ratio (left) and standard deviation of vibration amplitude (right) over $R e_{h}$ of the hydrofoil case compared with Ausoni [19].

$$
R e_{h}=3.86 \times 10^{4} \quad R e_{h}=5.31 \times 10^{4}
$$
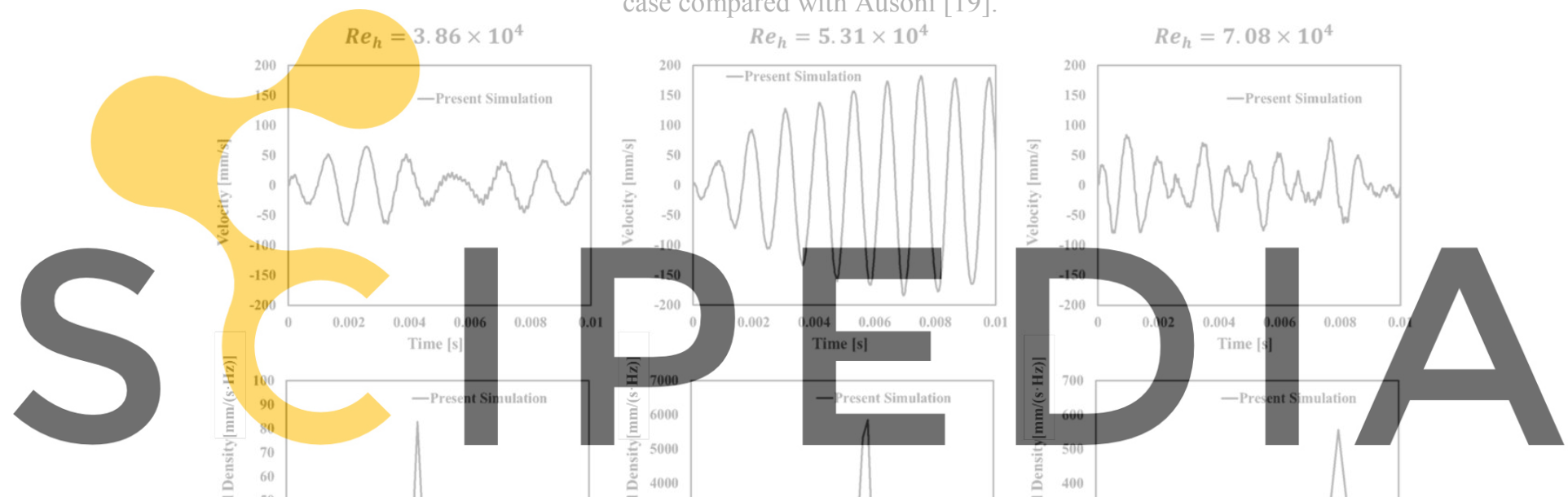

Register for free at http\$//www.scipe
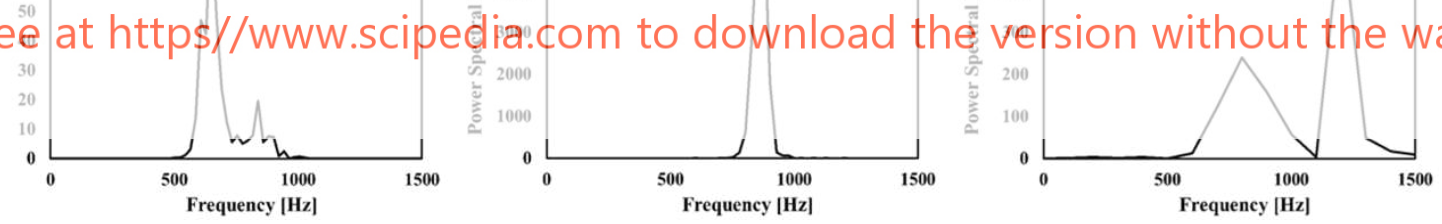

Figure 7: Instantaneous velocity magnitude (top) and spectra (bottom) of the hydrofoil for the 2-D simulations with three $R e_{h}$.

The frequency ratio is defined as the vortex shedding frequency $f_{s}$, to the natural frequency of the hydrofoil $\left(f_{n}\right)$, which is calculated by model analysis [12]. Figure 6 shows the frequency ratio and standard deviation of displacement amplitude at the hydrofoil surface at $80 \%$ chord length for different $R e_{h}$. Our results are compared against the experimental data of Ausoni [19]. Generally, the simulated both 2-D and 3-D frequency ratios agree well with the experimental results. When $R e_{h}=3.86 \times 10^{4}$, both $2-\mathrm{D}$ and 3 -D simulations give similar results. Differences between 2-D and 3-D simulations occur at the lock-in regime, in which a discontinuity also occur in the experimental data.

The structure's velocity magnitude at the hydrofoil surface at $80 \%$ chord length for three $R e_{h}$ are shown in Figure 7. The instantaneous hydrofoil's velocity at lock-in $\left(R e_{h}=5.31 \times\right.$ $10^{4}$ ) has significant features that distinguish it from other regimes: large amplitude with a single 
dominant frequency. These features are also shown in the velocity spectra plot of Figure 7 . The lock-in regime velocity has one dominant frequency with a large power spectral density, while the velocity in other regimes has two or more dominant frequencies with relatively small power spectral densities.

The pressure and velocity contours of the fluid domain at the time that the hydrofoil reaches its maximum displacement under the lock-in regime are shown in the top of Figure 8. It can be seen that vortex shedding in the wake is captured. The stress distribution and amplified displacement at the time that it reaches its maximum displacement under the lock-in regime is shown in the bottom of Figure 8.

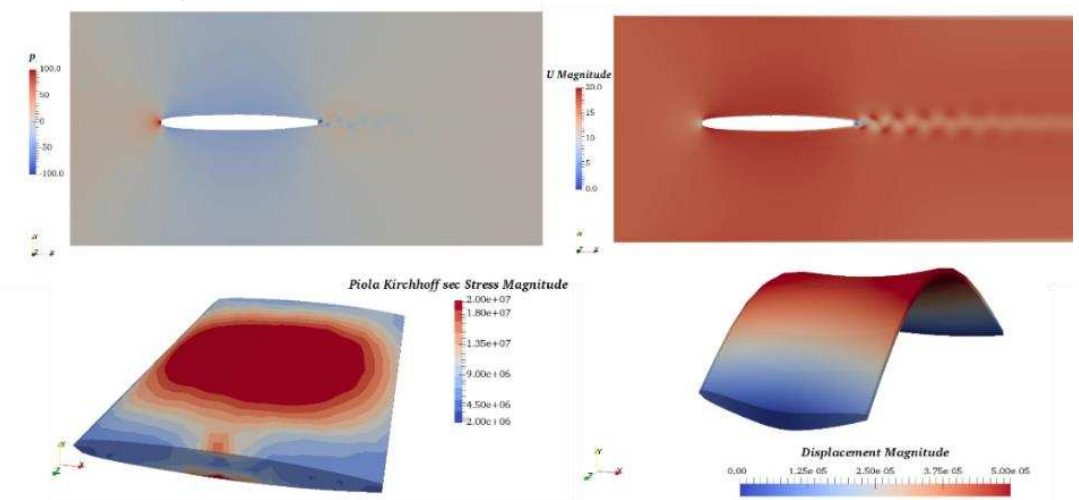

Figure 8: Pressure (top left), velocity (top right), stress (bottom left) and displacement contours with magnification 1,000 (bottom right) of the hydrofoil at its maximum displacement under the lock-in regime.

\section{CONCLUSIONS AND FUTURE WORK}

We have developed a framework for fluid-structure interaction simulations using a partitioned approach. Solvers are developed based on the open-source OpenFOAM and FEniCS frameworks are adopted as the CFD and CSM solvers, respectively. The open-source Multiscale Universal Interface (MUI) coupling library is employed as the interface between fluid and structural domains. Two FSI coupling algorithms, the Fixed Relaxation approach and the Aitken's $\delta^{2}$ approach, have been implemented within the MUI library to achieve a tight and stable coupling. Three validation cases are presented and compared with published results. These show the implementation to provide expected results. The vortex-shedding induced vibration of a hydrofoil is also presented. A comparison between the numerical simulation and the experimental data shows the capability of the present simulation framework to undertake challenging FSI simulations with a significant added mass effect. Current parallel scalability of the framework is also briefly discussed. This is currently under active development with a view towards an exascale capability. There is also an intention to include additional capability into the framework to satisfy different simulation needs, for example the inclusion of a meshless CFD approach.

\section{ACKNOWLEDGEMENTS}

This work was supported by the STFC Hartree Centre's Innovation Return on Research programme, funded by the Department for Business, Energy \& Industrial Strategy. Results were obtained using the Scafell Pike High-Performance Computer based at the STFC Hartree Centre at Daresbury Laboratory. We thank Mr Eduardo Ramos Fernandez and Dr Robert Sawko of 
IBM Research UK for their development of a Python wrapper for the MUI library.

\section{REFERENCES}

[1] Cajas, J.C., Houzeaux, G., Vazquez, M., García, M., Casoni, E., Calmet, H., Artigues, A., Borrell, R., Lehmkuhl, O. and Pastrana, D., Fluid-structure interaction based on HPC multicode coupling. SIAM Journal on Scientific Computing, 2018. 40(6): p. C677-C703.

[2] Houzeaux, G., Garcia-Gasulla, M., Cajas, J., Borrell, R., Santiago, A., Moulinec, C. and Vázquez, M., Parallel Multiphysics Coupling: Algorithmic and Computational Performances. International Journal of Computational Fluid Dynamics, 2020. 34(7-8): p. 486-507.

[3] Michler, C., Hulshoff, S., Van Brummelen, E. and De Borst, R., A monolithic approach to fluid-structure interaction. Computers \& fluids, 2004. 33(5-6): p. 839-848.

[4] Weller, H.G., Tabor, G., Jasak, H. and Fureby, C., A tensorial approach to computational continuum mechanics using object-oriented techniques. Computers in physics, 1998. 12(6): p. 620-631.

[5] Alnæs, M., Blechta, J., Hake, J., Johansson, A., Kehlet, B., Logg, A., Richardson, C., Ring, J., Rognes, M.E. and Wells, G.N., The FEniCS project version 1.5. Archive of Numerical Software, 2015. 3(100).

[6] Tang, Y.-H., Kudo, S., Bian, X., Li, Z. and Karniadakis, G.E., Multiscale universal interface: a concurrent framework for coupling heterogeneous solvers. Journal of Computational Physics, 2015. 297: p. 13-31.

[7] Skillen, A., Longshaw, S., Cartland-Glover, G., Moulinec, C. and Emerson, D., Profiling and application of the multi-scale universal interface (MUI), in 6th European Conference on Computational Mechanics (ECCM 6). 2018: Glasgow.

[8] Holzmann, T., Mathematics, numerics, derivations and OpenFOAM®. Loeben, Germany: Holzmann CFD, 2016.

[9] Jasak, H. and Tukovic, Z., Automatic mesh motion for the unstructured finite volume method. Transactions of FAMENA, 2006. 30(2): p. 1-20.

[10] Slyngstad, A.S., Verification and Validation of a Monolithic Fluid-Structure Interaction Solver in FEniCS. A comparison of mesh lifting operators. 2017.

[11] Razzaq, M., Turek, S., Hron, J., Acker, J., Weichert, F., Grunwald, I., Roth, C., Wagner, M. and Romeike_, B., Numerical simulation and benchmarking of fluid-structure interaction with application to hemodynamics, in Fundamental Trends in Fluid-Structure Interaction. 2010, World Scientific. p. 171199.

[12] Bleyer, J., Numerical tours of computational mechanics with FEniCS. Zenodo, 2018.

[13] Erlicher, S., Bonaventura, L. and Bursi, O.S., The analysis of the generalized- $\alpha$ method for non-linear dynamic problems. Computational Mechanics, 2002. 28(2): p. 83-104.

[14] Rendall, T. and Allen, C., Improved radial basis function fluid-structure coupling via efficient localized implementation. International journal for numerical methods in engineering, 2009. 78(10): p. 1188-1208.

[15] Liu, W., Wang, W., Skillen, A., Fernandez, E.R., Longshaw, S. and Sawko, R., Code Development on Parallel Partitioned Fluid-Structure Interaction Simulations, Technical Report. 2020.

[16] Turek, S. and Hron, J., Proposal for numerical benchmarking of fluid-structure interaction between an elastic object and laminar incompressible flow, in Fluid-structure interaction. 2006, Springer. p. 371 385.

[17] Tuković, Ž., Karač, A., Cardiff, P., Jasak, H. and Ivanković, A., OpenFOAM finite volume solver for fluid-solid interaction. Transactions of FAMENA, 2018. 42(3): p. 1-31.

[18] Richter, T., Goal-oriented error estimation for fluid-structure interaction problems. Computer Methods in Applied Mechanics and Engineering, 2012. 223: p. 28-42.

[19] Ausoni, P., Turbulent vortex shedding from a blunt trailing edge hydrofoil, PhD Thesis. 2009, EPFL.

[20] Menter, F.R., Influence of freestream values on k-omega turbulence model predictions. AIAA journal, 1992. 30(6): p. 1657-1659. 